\title{
Effect of bisphosphonate on temporomandibular joint in osteopenia- induced rats by botulinum toxin $A$ injection on masticatory muscle: a preliminary study
}

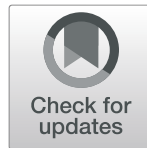

Jae-Young Kim, Dae-Hoon Kim, Hyo-Won Jang, Kwang-Ho Park and Jong-Ki Huh*

\begin{abstract}
Background: Botulinum toxin injection on the masticatory muscle induces the osteopenic condition on the ipsilateral condyle. Bisphosphonate suppresses bone resorption and is used to treat osteopenic or osteoporotic condition. This study aimed to evaluate the effect of bisphosphonate administration on prevention of condylar resorption and botulinum toxin A-induced disuse osteopenia in rats.

Results: The volume of the condyle and bone volume/tissue volume (BV/TV, \%) showed a strong tendency towards statistical significance ( $p=0.052$ and 0.058). Trabecular thickness (Tb.Th, mm) and trabecular number (Tb.N, $1 / \mathrm{mm}$ ) were significantly smaller in the Botox group than in the other groups $(p<0.05)$. The volume of the condyle and BV/TV in the bisphosphonate 100 and bisphosphonate 200 groups showed similar values when compared with the control group.

Conclusion: Bisphosphonate administration after botulinum toxin A injection in the masticatory muscles appears to prevent condyle resorption and botulinum toxin-induced disuse osteopenia in rats.
\end{abstract}

Keywords: Bisphosphonate, Botulinum toxin, Mandibular condyle, Temporomandibular joint

\section{Background}

Botulinum toxin causes transient paralysis of muscles by blocking acetylcholine release at the neuromuscular junction [1]. Botulinum toxin A (BTX-A) is most widely used among the seven types of toxins produced by Clostridium species [2]. BTX-A is used for cosmetic purpose in patients with masseter muscle hypertrophy [3]. In addition, BTX-A is used for reducing pain in temporomandibular disorders [4].

Tsai et al. reported that a decrease in muscle volume leads to a decrease in mandibular bone mass [5]. An experimental study on rabbits reported significant decrease in bone quality and quantity in the condylar head at 4 weeks after botulinum toxin injection (BTI). Although functional parameters reverted to near pre-injection

\footnotetext{
* Correspondence: omshuh@yuhs.ac

Department of Oral and Maxillofacial Surgery, Gangnam Severance Hospital,

Yonsei University College of Dentistry, 211 Eonju-ro, Gangnam-gu, Seoul 06273, South Korea
}

\section{Springer Open}

(c) The Author(s). 2019 Open Access This article is distributed under the terms of the Creative Commons Attribution 4.0 International License (http://creativecommons.org/licenses/by/4.0/), which permits unrestricted use, distribution, and reproduction in any medium, provided you give appropriate credit to the original author(s) and the source, provide a link to the Creative Commons license, and indicate if changes were made. remained atrophic and percentage of bone area in the condylar head was low [6].

Bisphosphonates (BPs) suppress bone resorption through various mechanisms such as decrease in turnover rate, inhibition of activity, and induction of apoptosis of osteoclasts $[7,8]$. In this regard, BPs have been used for the prevention of osteoporosis, bone metastasis of malignant tumor, and pathologic fracture.

Thus, we hypothesized that the application of bisphosphonate after BTX-A (injected to unilateral masticatory muscles) could contribute to the inhibition of bone resorption or osteopenic condition of ipsilateral condylar through inhibition of osteoclast action. The purpose of this study was to evaluate the effect of bisphosphonate administration on the prevention of condylar resorption and botulinum toxin A-induced disuse osteopenia in rats. 


\section{Methods}

\section{Animal model and study design (Fig. 1)}

Sixteen female Sprague-Dawley (SD) rats (Orientbio Co. Ltd., Seongnam-si, Gyenggi-do, Korea) were used in the present study. All rats were 10-week-old of age. Two rats were housed per cage and were individually marked. The cages were placed in a room with filtered air at a temperature of $22{ }^{\circ} \mathrm{C} \pm 2{ }^{\circ} \mathrm{C}$ and $50 \% \pm 10 \%$ relative humidity. A 12-h light/dark cycle was maintained. The animals were fed a normal rodent diet and water ad libitum. Animals were acclimated for 1 week prior to the beginning of the study. This study was approved by and performed in accordance with the guidelines of the institutional animal research ethics committee (IACUC No. 2017-0083).

The animals were randomly divided into four groups: control, Botox (BTX), bisphosphonate 100 (BP100), and bisphosphonate 200 (BP200) $(n=4)$. In the first and third week, $3.75 \mathrm{U}$ of botulinum toxin injection (BTI) (Botulax ${ }^{\oplus}$, Botulinum toxin A, HUGEL, Chuncheon, Korea) was administered on the left side of the masseter $(2.5 \mathrm{U})$ and temporalis muscles $(1.25 \mathrm{U})$ in all groups except the control group under general anesthesia with isoflurane. In the control group, same dosage of normal saline was administered on the left side of the masseter and temporalis muscles.

A total of $100 \mu \mathrm{g} / \mathrm{kg}$ (for BP100 group) or $200 \mu \mathrm{g} / \mathrm{kg}$ (for BP200 group) of zoledronic acid (ZA, Zometa ready $^{\oplus}$, Novartis, Switzerland) was injected into the peritoneum, for 2 weeks after injection of BTI (in fourth and fifth week of experiment). There was a 1-week waiting period (sixth week) for the effect of administered bisphosphonate. All the animals were euthanized when the seventh week of experiment began.

\section{Microscopic computed tomography}

Microscopic computed tomographic ( $\mu$ CT, Skyscan 1173, Kontich, Belgium) images were scanned at $10.65-\mu \mathrm{m}$ pixel size at an energy level of $90 \mathrm{kV}$ and evaluated using CTAn ${ }^{\circ}$ software (Skyscan, Kontich, Belgium).

The region of interest (ROI) was focused between 0.53 $\mathrm{mm}$ and $1.28 \mathrm{~mm}$ from the most posterior margin of the condyle. Then, a $0.32 \mathrm{~mm} \times 0.32-\mathrm{mm}$ sized circle was set at the center of condyle. Finally, cylindrical shape was formed at the condyle area $(0.32 \mathrm{~mm}$ diameter and 0.75 $\mathrm{mm}$ height), and this area was analyzed (Fig. 2a, b). Bone volume/tissue volume $\left(\mathrm{BV} / \mathrm{TV}, \mathrm{mm}^{3}\right)$, trabecular numbers (Tb.N, 1/mm), trabecular thickness (Tb.Th, mm), and trabecular separation (Tb.Sp, $\mathrm{mm}$ ) of each group were calculated by $\mathrm{CTAn}^{\oplus}$ software.

Three-dimensional image reconstruction and analysis (Fig. 3) $\mu \mathrm{CT}$ images were converted to the Digital Imaging and Communications in Medicine (DICOM) files using a converting software (DicomCT ver 2.0, Bruker ${ }^{\circ}$ ). The 3D images were then reconstructed and analyzed using image analysis software (Mimics Research 21.0, Materialise, Belgium). The threshold was set between -500 and 750 Hounsfield Unit.

The reference points on the $3 \mathrm{D}$ images were defined as follows:

(1) Sigmoid notch (P1) - the lowest point of the sigmoid notch.

(2) Mandibular foramen (P2) - the most anterior point of the opening of the inferior alveolar nerve canal.

(3) Condyle superior (P3) - the most superior point of the condyle.

(4) Condyle anterior (P4) - the most anterior point of the condyle.

(5) Condyle posterior (P5) - the most posterior point of the condyle.

(6) Angle (P6) - the most prominent point of the mandibular angle.

The reference planes on the 3D images were defined as follows:

(1) Sagittal plane (plane S) - a plane passing through P1, P2, and P6.

(2) Coronal plane (plane C) - a plane normal to the plane S passing through P1 and P6.

\begin{tabular}{|c|c|c|c|c|c|c|c|}
\hline & Week 1 & Week 2 & Week 3 & Week 4 & Week 5 & Week 6 & Week 7 \\
\hline Control & Saline* & Waiting & Saline* & Saline $\dagger$ & Salinet & Waiting & Euthanasia \\
\hline BTX & BTI & Waiting & BTI & Saline $\dagger$ & Saline $\dagger$ & Waiting & Euthanasia \\
\hline BP100 & BTI & Waiting & B & BP100 & BP100 & Waiting & Euthanasia \\
\hline BP200 & BTI & Waiting & & BP200 & BP200 & Waiting & Euthanasia \\
\hline
\end{tabular}

Fig. 1 Experiment design. $3.75 \mathrm{U}$ of botulinum toxin and the same dose of normal saline were administered in weeks 1 and 3 . In weeks 4 and 5 , $100 \mu \mathrm{g} / \mathrm{kg}$ or $200 \mu \mathrm{g} / \mathrm{kg}$ of bisphosphonate (zoledronic acid) was administered to the respective groups. A total of $100 \mu \mathrm{g} / \mathrm{kg}$ of normal saline was administered to both the control and BTX groups. All animals were euthanized on week 7 

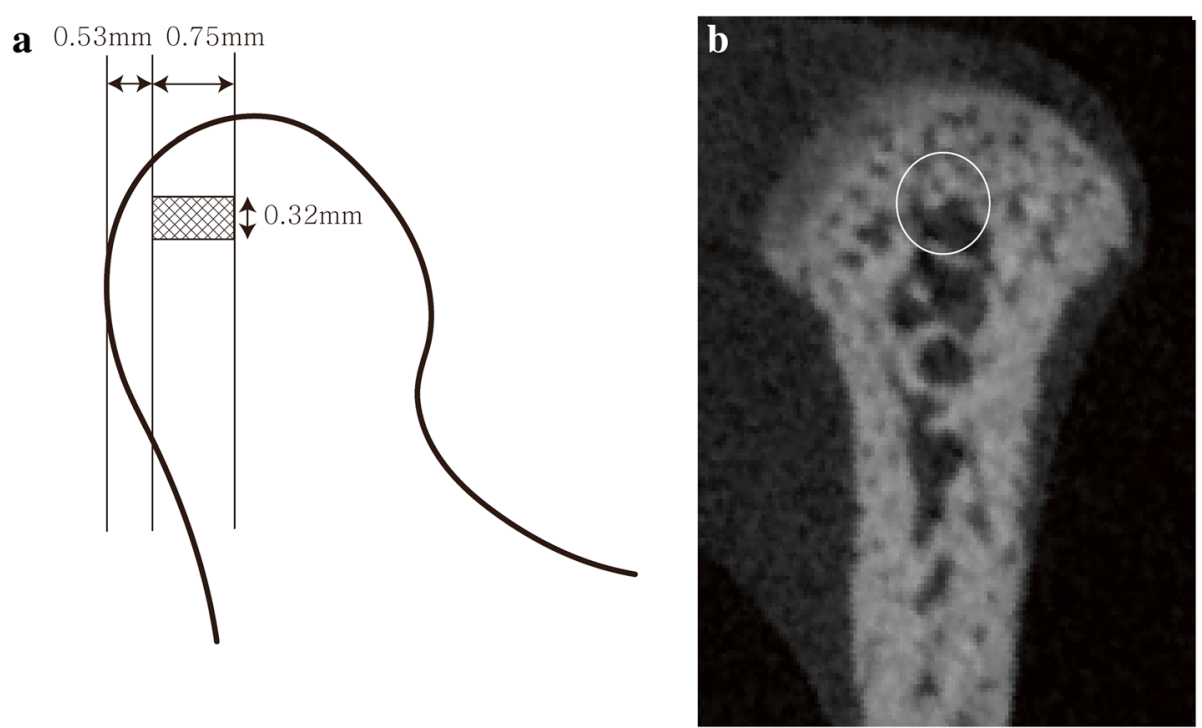

Fig. 2 Range of analysis (a) and region of interest (ROI) (b). The region of interest (ROI) was focused between 0.53 and $1.28 \mathrm{~mm}$ from the most posterior margin of the condyle. Then, a $0.32 \mathrm{~mm} \times 0.32 \mathrm{~mm}$ sized circle was set at the center of the condyle. Finally, cylindrical shape was formed at the condyle area $(0.32 \mathrm{~mm}$ diameter and $0.75 \mathrm{~mm}$ height), and this area was analyzed

The volume of condyle (V_Con) is measured above the plane $\mathrm{S}$, which was calculated automatically by the analysis software (Fig. 3). Distance and angle from P1 and P2 to each point on the condyle (P3, P4, and P5) were measured, the values of which are summarized in Table 1.

\section{Statistical analyses}

The data were analyzed using a statistical analysis program (SPSS 23.0, IBM, USA). $\mu \mathrm{CT}$, the volume, and linear and angular values were compared using Kruskal-Wallis analysis. $p$ values $<0.05$ indicated as statistically significant.

\section{Results}

One rat in the BTX and BP100 group died during the experiment. Therefore, the analysis was performed on three rats in the BTX and BP100 group and four rats in the other groups.

\section{$\mu \mathrm{CT}$ analysis (Table 2)}

The BTX group had the lowest BV/TV (\%), with marginal trend towards significance when compared to the other groups $(p=0.052)$. However, other groups showed similar values. Tb.Th $(\mathrm{mm})$ and Tb.N $(1 / \mathrm{mm})$ values

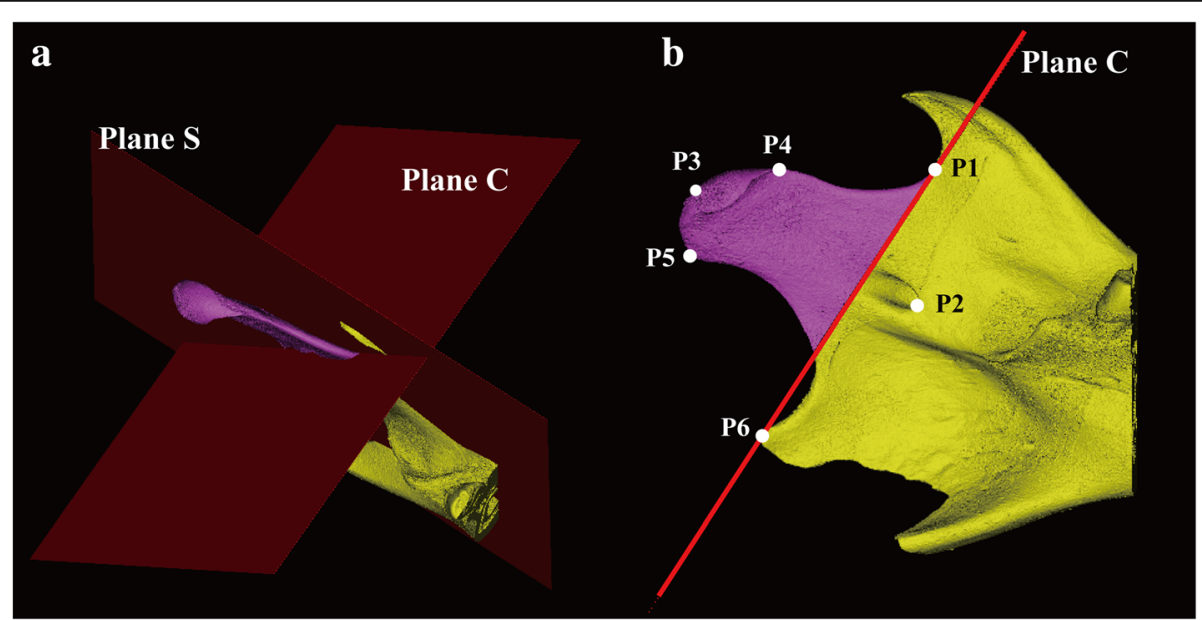

Fig. 3 Reference planes (a) and reference points (b). Plane S refers to a plane passing through P1, P2, and P6. Plane C refers to a plane normal to plane $\mathrm{S}$, passing through $\mathrm{P} 1$ and $\mathrm{P} 6$. The condyle component was separated by plane $\mathrm{C}$ from the other part of the mandible. P1, sigmoid notch; P2, mandibular foramen; P3, condyle superior; P4, condyle anterior; P5, condyle posterior; P6, angle 
Table 1 Definition of the volume of condyle angle and distance measurement

\begin{tabular}{ll}
\hline Measurements & Definition \\
\hline V_Con $\left(\mathrm{mm}^{3}\right)$ & Volume of condyle calculated above plane C \\
Ang123 $(\mathrm{deg})$ & Angle between P1, P2, and P3 \\
Ang124 (deg) & Angle between P1, P2, and P4 \\
Ang125 (deg) & Angle between P1, P2, and P5 \\
D12 $(\mathrm{mm})$ & Distance between P1 and P2 \\
D13 $(\mathrm{mm})$ & Distance between P1 and P3 \\
D14 $(\mathrm{mm})$ & Distance between P1 and P4 \\
D15 $(\mathrm{mm})$ & Distance between P1 and P5 \\
D23 $(\mathrm{mm})$ & Distance between P2 and P3 \\
D24 $(\mathrm{mm})$ & Distance between P2 and P4 \\
D25 $(\mathrm{mm})$ & Distance between P2 and P5
\end{tabular}

$V \_$Con volume of condyle, Ang angle, $D$ distance, Plane $C$ coronal plane, $P 1$ sigmoid notch, $P 2$ mandibular foramen, $P 3$ condyle superior, $P 4$ condyle anterior, $P 5$ condyle posterior, $P 6$ angle

were the lowest in the BTX group with statistically significant difference $(p=0.025$ and $p=0.044$, respectively). Tb.Sp ( $\mathrm{mm})$ was the highest in the BTX group. However, no significant difference was observed among the four groups (Fig. 4a-d).

\section{Volumetric, linear, and angular measurement (Table 3)}

The volume of the condyle was the smallest in the BTX group $\left(7.68 \pm 0.19 \mathrm{~mm}^{3}\right)$, with at the edge of significance when compared to the other groups $(p=0.058)$. The condylar volumes were $10.44 \pm 0.89 \mathrm{~mm}^{3}, 10.58 \pm 1.02$ $\mathrm{mm}^{3}$, and $9.81 \pm 1.03 \mathrm{~mm}^{3}$ in the control, BP100, and BP200 groups, respectively (Fig. 4e).

Among D13, D14, D15, D23, D24, and D25 (the distance from P1 (sigmoid notch) or P2 (lingula) to P3 (condyle superior), P4 (condyle anterior), and P5 (condyle posterior)), only D14 and D24 showed a significant difference $(p<0.05)$.

The angle between P1, P2, and P4 (Ang124) was the largest in the BTX group $\left(56.79 \pm 6.80^{\circ}\right)$ and the smallest $\left(43.57 \pm 5.18^{\circ}\right)$ in the control group $(p=0.080)$.

\section{Discussion}

Botulinum toxin A (BTX-A) has been used for the treatment of masseter hypertrophy, masticatory myalgia, and temporomandibular joint disorders [3, 4, 9]. Injection of BTX-A into the masticatory muscles induces transient muscle paralysis, which may increase the risk of reduction in bone mineral density, or "disuse atrophy" [10]. Several studies have reported on the resorption and/or trabecular changes in the condyle [5, 9-11]. These changes may occur in both experimental conditions and clinical situations. In the present study, $3.75 \mathrm{U}$ of botulinum toxin $\mathrm{A}$ was injected in the left masseter muscle $(2.5 \mathrm{U})$ and left temporal muscle $(1.25 \mathrm{U})$ under general anesthesia with isoflurane based on the protocol reported in previous studies $[9,12]$.

Bisphosphonates suppress osteoclastic activity and induce apoptosis of osteoclasts, thereby exerting a protective effect on bone loss $[7,8,13]$. Several investigators reported that BPs prevented subchondral bone loss on articulation in experimental animal studies [14-16]. Chen et al. specifically reported that the injection of $\mathrm{BP}$ into the temporomandibular joint articulation could reduce changes in cartilage and subchondral bone loss [16].

In this regard, the hypothesis of the present study was that the administration of $\mathrm{BP}$ after botulinum toxin A injection into masticatory muscles has a protective potential in the maintenance of the volume of the condyle.

One study reported that the dose of bisphosphonate (zoledronate) administered to prevent bone metastasis in a patient with malignant tumor was $67 \mu \mathrm{g} / \mathrm{kg}$ every 4 weeks [17]. Black et al. reported an increase in bone mineral density on yearly infusion of $5 \mathrm{mg}$ zoledronic acid by intravenous route [18]. The dose amounts to approximately $7 \mu \mathrm{g} / \mathrm{kg} /$ week, when converted, in a $60 \mathrm{~kg}$ adult. In a study by Chen et al., which investigated the prevention of condyle resorption in rats, $100 \mu \mathrm{g}$ of alendronate was administered at weekly intervals for 4 weeks [16]. In the present study, $100 \mu \mathrm{g} / \mathrm{kg}$ (BP100 group) and $200 \mu \mathrm{g} / \mathrm{kg}$ (BP200 group) bisphosphonate were administered considering (1) relatively fast bone turnover rate in rats, (2) short-term dosing period (2 weeks), and (3) intraperitoneal route of administration.

As a result, the volume of the condyle showed similar values between the BP100 group (receiving $100 \mu \mathrm{g} / \mathrm{kg}$ ) and the BP200 group (receiving $200 \mu \mathrm{g} / \mathrm{kg}$ ). The volume of the condyle was the lowest in the BTX group with a strong tendency towards statistical significance when

Table 2 Microscopic computed tomography $(\mu \mathrm{CT})$ analysis of the left condyle

\begin{tabular}{|c|c|c|c|c|c|}
\hline & Control $(n=4)$ & $\operatorname{BTX}(n=3)$ & BP100 $(n=3)$ & BP200 $(n=4)$ & $p$ value \\
\hline BV/TV (\%) & $83.41 \pm 6.69$ & $42.55 \pm 8.94$ & $74.66 \pm 17.66$ & $72.23 \pm 11.87$ & 0.052 \\
\hline Tb.Th $(m m)^{*}$ & $0.12 \pm 0.02$ & $0.07 \pm 0.00$ & $0.09 \pm 0.02$ & $0.09 \pm 0.01$ & 0.025 \\
\hline Tb.N $(1 / \mathrm{mm})^{*}$ & $7.03 \pm 0.79$ & $6.50 \pm 1.05$ & $8.22 \pm 0.47$ & $8.07 \pm 0.87$ & 0.044 \\
\hline Tb.Sp (mm) & $0.06 \pm 0.01$ & $0.09 \pm 0.02$ & $0.06 \pm 0.02$ & $0.08 \pm 0.18$ & 0.102 \\
\hline
\end{tabular}

BTX botulinum toxin, BP100 $100 \mu \mathrm{g} / \mathrm{kg}$ of bisphosphonate, BP200 $200 \mu \mathrm{g} / \mathrm{kg}$ of bisphosphonate, BV/TV bone volume/tissue volume, $T b . T h$ trabecular thickness, $T b . N$ trabecular number, $T b . S p$ trabecular separation

*Significant difference $(p<0.05)$, mean \pm SD 

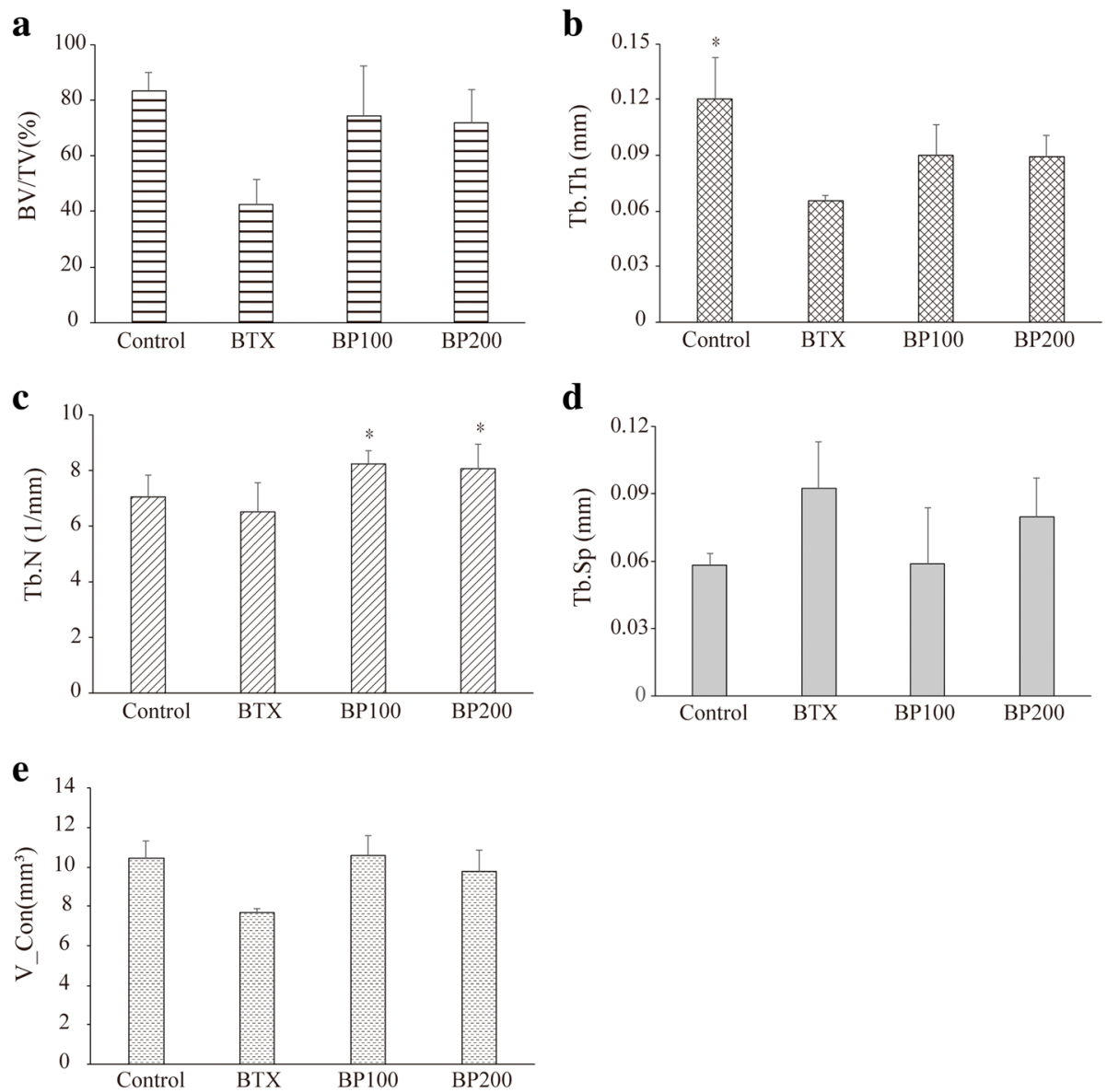

Fig. 4 Microscopic computed tomography $(\mu \mathrm{CT})$ analysis $(\mathbf{a}-\mathbf{d})$ and three-dimensional analysis of condylar volume (e). a Bone volume/tissue volume (BV/TV, \%) in the BTX group was the lowest with marginal trend towards significance when compared to the other groups $(p=0.052)$. $\mathbf{b}$ Trabecular thickness (Tb.Th, $\mathrm{mm}$ ) was the lowest in the BTX group $(p=0.025)$. However, significant difference was observed only between the BTX and control groups. c Trabecular number (Tb.N, 1/mm) was the lowest in BTX. BP100 and BP200 groups showed significant higher value compared with the BTX group. d Trabecular number (Tb.N, 1/mm) and trabecular separation (Tb.Sp, mm) showed no significant difference among the four groups. e Volume of the condyle on three-dimensional analysis showed similar result with BV/TV (\%). *Statistically significant difference compared with the BTX group $(p<0.05)$

Table 3 Volumetric, angular, and linear analysis of the left side of the mandible

\begin{tabular}{|c|c|c|c|c|c|}
\hline & Control $(n=4)$ & BTX $(n=3)$ & BP100 $(n=3)$ & BP200 $(n=4)$ & $p$ value \\
\hline $\bar{V}$ _Con $\left(\mathrm{mm}^{3}\right)$ & $10.44 \pm 0.89$ & $7.68 \pm 0.19$ & $10.58 \pm 1.02$ & $9.81 \pm 1.03$ & 0.058 \\
\hline Ang123 (deg) & $63.14 \pm 3.33$ & $70.72 \pm 5.07$ & $67.86 \pm 5.29$ & $61.87 \pm 4.57$ & 0.123 \\
\hline Ang124 (deg) & $43.57 \pm 5.18$ & $56.79 \pm 6.80$ & $53.01 \pm 4.41$ & $47.09 \pm 4.82$ & 0.080 \\
\hline Ang125 (deg) & $80.93 \pm 5.62$ & $84.71 \pm 6.31$ & $87.23 \pm 6.00$ & $80.48 \pm 4.26$ & 0.473 \\
\hline $\mathrm{D} 12$ (mm) & $3.98 \pm 0.08$ & $3.86 \pm 0.33$ & $3.67 \pm 0.30$ & $3.83 \pm 0.17$ & 0.454 \\
\hline $\mathrm{D} 13$ (mm) & $6.57 \pm 0.07$ & $7.10 \pm 0.54$ & $7.00 \pm 0.30$ & $6.58 \pm 0.23$ & 0.073 \\
\hline $\mathrm{D} 14(\mathrm{~mm})^{*}$ & $3.66 \pm 0.40$ & $5.11 \pm 0.57$ & $4.74 \pm 0.22$ & $4.34 \pm 0.32$ & 0.014 \\
\hline D15 (mm) & $7.14 \pm 0.18$ & $7.72 \pm 0.48$ & $7.56 \pm 0.37$ & $7.33 \pm 0.35$ & 0.158 \\
\hline $\mathrm{D} 23(\mathrm{~mm})$ & $7.33 \pm 0.31$ & $7.34 \pm 0.24$ & $7.49 \pm 0.12$ & $7.44 \pm 0.10$ & 0.564 \\
\hline $\mathrm{D} 24(\mathrm{~mm})^{*}$ & $5.30 \pm 0.21$ & $6.04 \pm 0.12$ & $5.92 \pm 0.18$ & $5.92 \pm 0.07$ & 0.026 \\
\hline $\mathrm{D} 25(\mathrm{~mm})$ & $6.60 \pm 0.29$ & $7.03 \pm 0.10$ & $6.78 \pm 0.09$ & $6.92 \pm 0.21$ & 0.064 \\
\hline
\end{tabular}


compared with other groups $(p=0.058)$. In the $\mu \mathrm{CT}$ analysis, the BV/TV of BTX group also showed the lowest value with a strong tendency towards statistical significance compared with the other three groups $(p=0.052)$.

Both cortical bone thickness and trabecular bone area decreased after the injection of botulinum toxin into the masticatory muscles $[1,5]$. Kün-Darbois et al. also reported a significant decrease in bone area/tissue area (B.Ar/T.Ar) on trabecular bone after the injection of botulinum toxin compared with the control group in $\mu C T$ analysis [9]. Recently, Vegger et al. reported that injection of $100 \mu \mathrm{g} / \mathrm{kg}$ zoledronic acid via the subcutaneous route prevented disuse osteopenia induced by botulinum toxin. Although the study focused on the limb and not the condyle, our group hypothesizes a similar effect on the temporomandibular joint [19].

Further studies with larger sample sizes are required to establish the preventive effect of bisphosphonate on disuse osteopenia and condylar resorption.

\section{Conclusion}

The result of the present study suggests that bisphosphonate administration after botulinum toxin A injection into the masticatory muscle may have a preventive effect on condyle resorption and botulinum toxin-induced disuse osteopenia in rats. However, the finding of this study is preliminary and requires further evaluation.

\begin{abstract}
Abbreviations
B.Ar/T.Ar: Bone area/tissue area; BP: Bisphosphonate; BTI: Botulinum toxin injection; BTX-A: Botulinum toxin A; BV/TV: Bone volume/tissue volume; DICOM: Digital Imaging and Communications in Medicine; ROI: Region of interest; Tb.N: Trabecular numbers; Tb.Sp: Trabecular separation;

Tb.Th: Trabecular thickness; $\mu \mathrm{CT}$ : Microscopic computed tomography
\end{abstract}

\section{Acknowledgements}

We would like to thank Hye Sun Lee (Ph.D., Biostatistician) and Sinae Kim (M.S. Biostatistician) of the Biostatistics Collaboration Unit, Yonsei University College of Medicine, Seoul, Korea, for their help with the statistical analyses.

\section{Funding}

This study was supported by a faculty research grant from the Yonsei University College of Dentistry (6-2015-0112).

\section{Availability of data and materials}

The datasets used and/or analyzed during the current study are available from the corresponding author on reasonable request.

\section{Authors' contributions}

JYK designed the experiment and prepared the manuscript. DHK and HWJ conducted the experiment. KHP designed the experiment. JKH designed and confirmed the experiment and revised the manuscript as the corresponding author. All authors read and approved the final manuscript.

\section{Ethics approval and consent to participate}

This study was approved by and performed in accordance with the guidelines of the institutional animal research ethics committee (IACUC No. 2017-0083).

\section{Consent for publication}

Not applicable

\section{Competing interests}

The authors declare that they have no competing interests.

\section{Publisher's Note}

Springer Nature remains neutral with regard to jurisdictional claims in published maps and institutional affiliations.

Received: 1 January 2019 Accepted: 15 February 2019

Published online: 04 March 2019

\section{References}

1. Matthys T, Ho Dang HA, Rafferty KL, Herring SW (2015) Bone and cartilage changes in rabbit mandibular condyles after 1 injection of botulinum toxin. Am J Orthod Dentofac Orthop 148:999-1009

2. Davletov B, Bajohrs M, Binz T (2005) Beyond BOTOX: advantages and limitations of individual botulinum neurotoxins. Trends Neurosci 28:446-452

3. Kim NH, Park RH, Park JB (2010) Botulinum toxin type A for the treatment of hypertrophy of the masseter muscle. Plast Reconstr Surg 125:1693-1705

4. Ernberg M, Hedenberg-Magnusson B, List T, Svensson P (2011) Efficacy of botulinum toxin type $\mathrm{A}$ for treatment of persistent myofascial TMD pain: a randomized, controlled, double-blind multicenter study. Pain 152:1988-1996

5. Tsai CY, Huang RY, Lee CM, Hsiao WT, Yang LY (2010) Morphologic and bony structural changes in the mandible after a unilateral injection of botulinum neurotoxin in adult rats. J Oral Maxillofac Surg 68:1081-1087

6. Rafferty KL, Liu ZJ, Ye W et al (2012) Botulinum toxin in masticatory muscles: short- and long-term effects on muscle, bone, and craniofacial function in adult rabbits. Bone 50:651-662

7. Drake MT, Clarke BL, Khosla S (2008) Bisphosphonates: mechanism of action and role in clinical practice. Mayo Clin Proc 83:1032-1045

8. Khosla S, Bilezikian JP, Dempster DW et al (2012) Benefits and risks of bisphosphonate therapy for osteoporosis. J Clin Endocrinol Metab 97:22722282

9. Kün-Darbois JD, Libouban H, Chappard D (2015) Botulinum toxin in masticatory muscles of the adult rat induces bone loss at the condyle and alveolar regions of the mandible associated with a bone proliferation at a muscle enthesis. Bone 77:75-82

10. Raphael KG, Tadinada A, Bradshaw JM et al (2014) Osteopenic consequences of botulinum toxin injections in the masticatory muscles: a pilot study. J Oral Rehabil 41:555-563

11. Aziz J, Awal D, Ayliffe P (2017) Resorption of the mandibular condyle after injections of botulinum toxin A. Br J Oral Maxillofac Surg 55:987-988

12. Seok H, Kim SG, Kim MK, Jang I, Ahn J (2018) Effect of the masseter muscle injection of botulinum toxin $\mathrm{A}$ on the mandibular bone growth of developmental rats. Maxillofac Plast Reconstr Surg 40:5

13. Allen MR (2008) Skeletal accumulation of bisphosphonates: implications for osteoporosis treatment. Expert Opin Drug Metab Toxicol 4:1371-1378

14. Shirai T, Kobayashi M, Nishitani $\mathrm{K}$ et al (2011) Chondroprotective effect of alendronate in a rabbit model of osteoarthritis. J Orthop Res 29:1572-1577

15. Zhu S, Chen K, Lan Y, Zhang N, Jiang R, Hu J (2013) Alendronate protects against articular cartilage erosion by inhibiting subchondral bone loss in ovariectomized rats. Bone 53:340-349

16. Chen K, Zhang N, Ding L, Zhang W, Hu J, Zhu S (2014) Early intra-articular injection of alendronate reduces cartilage changes and subchondral bone loss in rat temporomandibular joints after ovariectomy. Int J Oral Maxillofac Surg 43:996-1004

17. Kim JW, Cha IH, Kim SJ, Kim MR (2016) Biomarkers for bisphosphonaterelated osteonecrosis of the jaw. Clin Implant Dent Relat Res 18:281-291

18. Black DM, Delmas PD, Eastell R et al (2007) Once-yearly zoledronic acid for treatment of postmenopausal osteoporosis. N Engl J Med 356:1809-1822

19. Vegger JB, Brüel A, Thomsen JS (2018) Zoledronic acid prevents disuse osteopenia and augments gene expression of osteoclastic differentiation markers in mice. J Musculoskelet Neuronal Interact 18:165-175 